\title{
Elaboration de tarifs de cubage pour le genre Rhizophora au Delta du Saloum (Sénégal)
}

\author{
Ngor NDOUR ${ }^{1 *}$, Bienvenu SAMBOU ${ }^{2}$ et Maurice DASYLVA ${ }^{1}$ \\ ${ }^{1}$ Université Assane SECK de Ziguinchor, Département d'Agroforesterie, Sénégal. \\ ${ }^{2}$ Université Cheikh Anta Diop, Institut des Sciences de l'Environnement, Dakar, Sénégal. \\ *Auteur correspondant ; E-Mail : ngorndour@univ-zig.sn; BP : 523, Tél: + 2215533203
}

\section{RESUME}

Au Delta du Saloum, les populations habitent dans la mangrove où elles exploitent les ressources halieutiques et ligneuses depuis des siècles. Cependant, la croissance démographique notée dans la zone a contribué à l'augmentation des besoins en bois de la population. En plus de l'accroissement des besoins énergétiques, la superficie de la mangrove a diminué ces dernières années au Saloum. Dans l'optique de connaître le volume de bois de mangrove disponible et de mieux gérer la ressource, l'étude s'est focalisée sur l'élaboration de tarifs de cubage à partir de trois modèles d'ajustement théoriques. En termes de résultats, six tarifs ont été élaborés pour le genre Rhizophora. Parmi eux, deux tarifs $\left(\mathrm{Y}_{2}\right.$ et $\left.\mathrm{Y}_{4}\right)$ sont les plus pertinents pour le cubage des tiges de palétuviers. Leurs coefficients de détermination $\left(\mathrm{R}^{2}\right)$, compris entre 0,956 et 0,968 , dénotent leur pertinence. En référence à leur intervalle de validité, $\mathrm{Y}_{2}$ est plus précis que $\mathrm{Y}_{4}$. Néanmoins, l'étude recommande l'utilisation de ces deux régressions pour la détermination du volume de bois de la mangrove du Delta du Saloum.

(C) 2014 International Formulae Group. All rights reserved.

Mots clés : Bois, besoins, population, volume, tarifs, pertinence, validité.

\section{INTRODUCTION}

Les forêts de mangrove représentent une importante source de devise pour le Sénégal. La pêche artisanale qui s'y déroule procure à l'Etat plus de 163,66 milliards de $\mathrm{F}$ CFA en valeur absolue, soit $4,08 \%$ en pourcentage du PIB. Par ailleurs, la filière contribue à hauteur de $13,70 \%$ et de $3,3 \%$ respectivement dans les secteurs primaire et tertiaire (Dione et al., 2005). Cependant, les mangroves régressent de plus en plus dans le pays, particulièrement au Delta du Saloum où les pertes de superficies sont importantes selon divers auteurs (Soumaré, 1992 ; Diop et al., 1997 ; Ndour, 2005 ; Faye et al., 2008 ; Balla et al., 2013). Cette régression de la mangrove est inquiétante d'autant plus que les besoins des populations en bois augmentent de plus en plus au Delta du Saloum (Pirard et al., 2002 ; Dia, 2003). Selon Ndiongue (2003), ils sont estimés à 157950 tonnes de bois pour le fumage du poisson en une campagne de 8 mois dans les communautés rurales de Djirnda et de Bassoul (Ndiongue, 2003). La consommation moyenne de bois mort est de $14,63 \mathrm{~kg} / \mathrm{jour}$ par boulanger et de $0,25 \mathrm{~kg}$ par personne et par repas pour le village de Baout dans la CR de Djirnda (Leruse, 2000). En tout 
état de cause, il serait inutile d'interdire aux populations de couper le bois de mangrove, étant donné que celui de la terre ferme leur ait difficilement accessible. D'ailleurs, ce bois se trouve dans le terroir d'autres populations ou dans le domaine classé. Il est donc indispensable de mieux valoriser la ressource à l'aide d'outils de gestion appropriés. A cet effet, la présente étude vise l'élaboration de tarifs de cubage pour l'évaluation rapide de la biomasse volumique des palétuviers. Ces outils devraient permettre aux agents des eaux et forêts et des parcs nationaux de quantifier le volume de bois disponible dans les écosystèmes de mangrove qu'ils gèrent. Disposant de cette information et connaissant les besoins en bois des populations, les agents de l'Etat devraient, dans leurs stratégies de gestion, octroyer des permis de coupe qui tiennent compte du capital ligneux de la mangrove du Delta du Saloum. Ainsi, l'Etat pourrait promouvoir l'aménagement forestier durable pour préserver la diversité de ses intérêts dans les écosystèmes de mangrove.

\section{MATERIEL ET METHODES}

\section{Choix des unités d'échantillonnage}

L'élaboration de tarifs de cubage pour les espèces du genre Rhizophora a nécessité le choix sélectif d'unités d'échantillonnage dans la Réserve de Biosphère du Delta du Saloum. Ce choix, basé sur les caractéristiques socioéconomiques et environnementales du milieu, a permis de diminuer la taille des échantillons et de réduire l'effort d'inventaire sur le terrain. Ainsi, quatre unités d'échantillonnage (U1, U2, U3 et U4) ont été choisies du Nord au Sud de la zone (Figure 1). Ce choix a été fait à l'aide de cartes topographiques et de photographies aériennes sous forme d'images panchromatiques. L'orientation dans la zone et le repérage des échantillons ont été possibles à l'aide d'un GPS (Global Positioning System) et d'une boussole Sylva. Les sites retrouvés sont marqués à la peinture blanche pour faciliter la collecte ultérieure des paramètres dendrométriques des palétuviers. Lors de la collecte des données, des scies à bois, pinces, sécateurs et coupe-coupe ont permis de procéder à la coupe rase des palétuviers dans des placettes de $2,5 \mathrm{~m}$ x 2,5 m. Le dispositif de collecte de données est un transect autour duquel sont placées alternativement des placettes d'inventaire. La distance parcourue entre deux placettes est choisie au hasard dans une série de longueurs comprises entre 5 et 35 m selon Ndour et al. (2013).

\section{Choix des tiges à cuber}

Le choix des tiges pour l'élaboration du tarif a tenu compte de l'étendue des unités d'échantillonnage, des sous unités à échantillonner et des difficultés à se déplacer dans la mangrove. Pour ces diverses raisons, le choix des tiges à cuber n'a pu se faire par la méthode classique qui consiste à les choisir sélectivement pour constituer un échantillon homogène et représentatif de la zone d'étude. A défaut d'adopter la méthode classique, les tiges de diamètre à la base supérieur ou égal à $3,5 \mathrm{~cm}$, de diamètre au fin bout supérieur ou égal à $3 \mathrm{~cm}$ et de longueur supérieurs ou égale à 2 mètres ont été mesurées pour les calculs de volume. Ce premier choix discriminatoire a été fait en tenant compte de la qualité de bois rond sur écorce et de la longueur moyenne des tiges que les populations prélèvent dans la mangrove pour le bois de feu, le fumage du poisson, le soutènement et la confection des charpentes de maisons.

\section{Mesures de volumes des tiges}

$\mathrm{Au}$ cours de la collecte des paramètres dendrométriques, lorsque la tige est ramifiée au-dessous de la base, les ramifications sont mesurées et considérées comme des tiges séparées. Lorsque la tige présente une anomalie à l'emplacement du niveau de mesure, les mesures de circonférence et de longueur sont prises légèrement au-dessus de la forme irrégulière (Dallmeier, 1992). Tenant compte de l'ensemble de ces critères de choix des tiges, leur volume sur pied abattu a été estimé à l'aide de la formule de Newton :

$$
V=\frac{L}{24 \pi}\left(c_{o}{ }^{2}+c_{f}{ }^{2}+4 c_{m}{ }^{2}\right)
$$


$\mathrm{C}_{0}=$ circonférence à $1 \mathrm{~cm}$ au-dessus des racines échasses

$\mathrm{C}_{\mathrm{f}}=$ circonférence au fin bout de la tige

$\mathrm{C}_{\mathrm{m}}=$ circonférence au milieu de la tige

$\mathrm{L}=$ longueur de la tige à cuber

Celle-ci a été choisie grâce à de son niveau de performance dans l'estimation du volume de différent corps de révolution selon Rondeux (1993).

\section{Traitement et analyse des données}

Les tiges, ayant satisfait aux critères dendrométriques, sont cubées par la formule de Newton à l'aide du progiciel Excel. Le prétraitement des données est basé sur une analyse statistique de la variation de la valeur moyenne du coefficient de forme des tiges \pm 2 fois la valeur de leur écart-type. $\mathrm{Ce}$ prétraitement qui a permis d'homogénéiser les échantillons de tiges par unité d'échantillonnage entre dans le cadre de l'apurement des données suggéré par Picard et al. (2012). Pour que les tarifs élaborés soient valables à l'échelle de la Réserve de Biosphère, les tiges issues de peuplements mono spécifiques ont été regroupées indépendamment des unités d'échantillonnage. Ainsi, l'échantillon homogène obtenu était de 125 tiges pour les peuplements de Rhizophora mangle L. de hauteur moyenne les plus représentatifs au Delta du Saloum. Celui constitué de $R$. racemosa/harisonnii, issu des deux types de strates, était de 107 tiges. Après avoir constitué les échantillons, l'élaboration des tarifs de cubage a nécessité l'identification d'ajustements déjà testés par divers auteurs comme Rondeux (1993) et Arsenault (1993). Ces ajustements sont : $Y_{1}=$ $\mathrm{a}_{\mathrm{o}}+\mathrm{a}_{1} \mathrm{C}_{0}{ }^{2} \mathrm{~L} ; \mathrm{Y}_{2}=\mathrm{a}_{\mathrm{o}}+\mathrm{a}_{1} \mathrm{C}_{0}{ }^{2}+\mathrm{a}_{2} \mathrm{C}_{0}{ }^{2} \mathrm{~L}$ et $\mathrm{Y}_{3}=$ $a_{0}+a_{1} C_{m}^{2} L$. Les constantes $\left(a_{0}, a_{1}\right.$ et $\left.a_{2}\right)$ sont les coefficients de régression partielle des ajustements. Ces coefficients ont été déterminés en traitant les données à l'aide du logiciel StatView5. La pertinence des ajustements obtenus est analysée en considérant leurs caractéristiques statistiques notamment les coefficients de détermination, de corrélation et la moyenne quadratique des résidus absolus $(\mathrm{Sa})$ et des résidus relatifs
(Sr). Le coefficient de variation résiduelle a été aussi déterminé par la formule suivante :

$\begin{aligned} C V R & =\frac{100 x \sigma_{v, x}}{\bar{v}} \\ \sigma_{v, x} & =\sqrt{\frac{1}{n-p-1} \sum_{i=1}^{n}(v i-v e)^{2}}\end{aligned}$

$v i$ est le volume mesuré et ve le volume estimé ;

$n$ est l'effectif des tiges et $p$ le nombre de variables explicatives ;

$\sigma_{\mathrm{v}, \mathrm{x}}$ est l'écart-type des résidus.

Les moyennes quadratiques des résidus absolus $(\mathrm{Sa})$ et relatifs $(\mathrm{Sr})$ qui sont de bons paramètres pour l'appréciation des ajustements (Palm, 1981 cité par Rondeux, 1993) ont été calculé à l'aide des formules suivantes :

$S_{a}=\sqrt{\frac{1}{n} \sum_{i=1}^{n}(v i-v e)^{2}}$

$S_{r}=\sqrt{\frac{1}{n} \sum_{i=1}^{n}\left(\frac{v i-v e}{v e}\right)^{2}}$

et

Dans le but d'examiner la précision des tarifs élaborés, leurs limites de validité ont été déterminées à l'aide de la formule $\bar{v} \pm \sigma$ avec $\bar{v}$ correspondant au volume moyen des tiges et $\sigma$ l'écart-type estimé des résidus. Ce dernier paramètre a été calculé pour chaque régression à l'aide de la formule suivante:

$$
\sigma_{v . x i \ldots x p}=\sqrt{\frac{1}{n-p-1} \sum_{i=1}^{n}\left(v_{i}-v e\right)^{2}}
$$

A cet égard, un tarif de cubage est précis lorsque $68 \%$ des tiges qui constituent l'échantillon ont leur volume mesuré dans l'intervalle $\bar{v} \pm \sigma \quad$ (Rondeux, 1993). Ce critère a permis de juger de la précision des tarifs élaborés. Les analyses statistiques ont été faites en utilisant le progiciel Excel et Staview5 pour le calcul des volumes, les analyses ANOVA et le test uni-varié. 


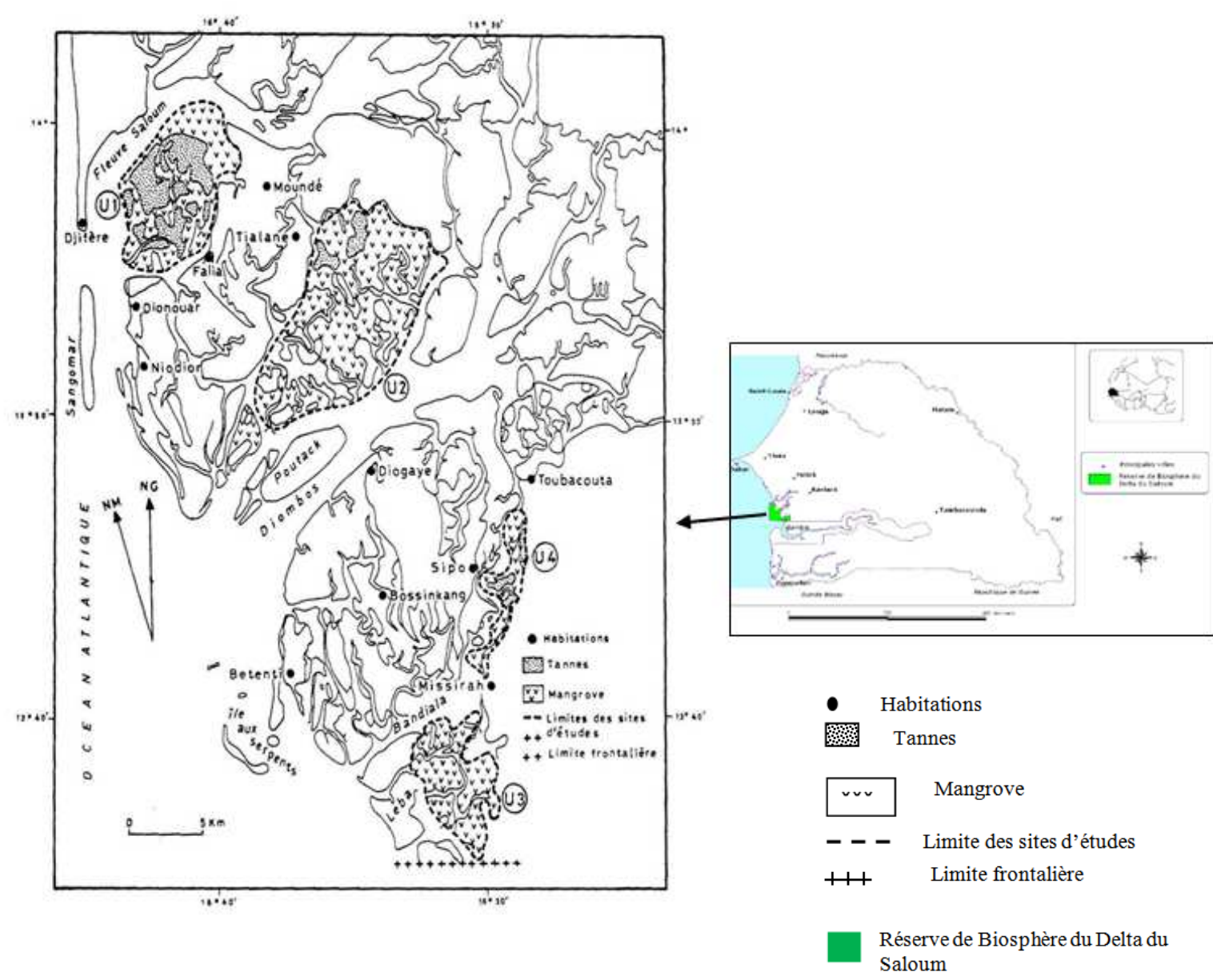

Figure 1 : Localisation des unités d'échantillonnage.

\section{RESULTATS}

L'échantillon homogène de 125 tiges de $R$. mangle a permis d'élaborer trois régressions pour la Réserve de Biosphère du Delta du Saloum. Les caractéristiques dendrométriques des tiges de l'échantillon sont consignées dans le Tableau 1. Il s'agit principalement de la longueur de la tige, sa circonférence à différentes hauteurs données et le volume correspondant.

\section{Tarifs de cubage de Rhizophora mangle}

Le traitement des données avec le logiciel Statview5 a permis de calculer les coefficients de régression partielle des trois tarifs de cubage. Leurs coefficients sont consignés dans le Tableau 2. Parmi eux, $\mathrm{a}_{0}$ est une valeur constante pour toutes les tiges à cuber alors que les coefficients $a_{1}$ et $a_{2}$ pondèrent la contribution des variables explicatives dans la détermination de la variable expliquée (volume). 
Le calcul de ces coefficients a permis d'établir la formule mathématique de chaque tarif de cubage. Ces tarifs de cubage individuel sont respectivement : $\mathrm{Y}_{1}=$ $0,001+0,045\left(\mathrm{C}_{0,1}\right)^{2} \mathrm{~L}$ avec $\mathrm{R}^{2}=0,965 ; \mathrm{Y}_{2}=$ $0,0004+0,045\left(\mathrm{C}_{0,1}\right)^{2}+0,037\left(\mathrm{C}_{0,1}\right){ }^{2} \mathrm{~L}$ avec $\mathrm{R}^{2}$ $=0,968$ et $\mathrm{Y}_{3}=0,0001+0,079\left(\mathrm{C}_{\mathrm{m}}\right){ }^{2} \mathrm{~L}$; avec $\mathrm{R}^{2}=0,993$. Chacun de ces tarifs permet de calculer le volume d'une tige en mètre cube.

\section{Analyse de la pertinence des tarifs de} cubage de Rhizophora mangle

Les paramètres statistiques considérés dans cette analyse sont les coefficients de détermination, de corrélation, de variation résiduelle et la moyenne quadratique des résidus absolus et relatifs de chaque régression. La valeur calculée de chacun de ces paramètres exprime le degré de pertinence de chaque régression. Les résultats obtenus sont consignés dans le Tableau 3. Les valeurs élevées des coefficients de détermination et de corrélation témoignent théoriquement d'une bonne régression. Celles du coefficient de variation résiduelle indiquent que la régression est pertinence lorsqu'elles sont faibles. Il en est de même pour les moyennes quadratiques des résidus absolus et résidus relatifs.

L'analyse des résultats montre que toutes les régressions élaborées sont pertinentes eu égard aux paramètres statistiques considérés. Les tarifs les plus pertinents sont respectivement $\mathrm{Y}_{3}, \mathrm{Y}_{2}$ et $\mathrm{Y}_{1}$ lorsque l'on tient compte de l'ensemble des paramètres statistiques.

\section{Analyse de la précision des tarifs élaborés pour $R$. mangle}

La détermination des limites de validité de la régression $\mathrm{Y}_{1}$ montre que celles-ci sont comprises dans l'intervalle [0,0068 - 0,011 $\mathrm{m}^{3}$ ]. Celles de $\mathrm{Y}_{2}$ et $\mathrm{Y}_{3}$ sont comprises respectivement dans les intervalles [0,003 -
$0,015]$ et $\left[0,008-0,010 \mathrm{~m}^{3}\right]$. Il ressort que seulement $22,4 \%$ des tiges de l'échantillon ont leur volume dans les limites de validité de $\mathrm{Y}_{1}$. Ce résultat signifie que $Y_{1}$ présente un petit intervalle de validité pour les mesures de volume. Dans le cas de la régression $\mathrm{Y}_{2}$, l'analyse des résultats montre que $72,8 \%$ des tiges ont leur volume dans l'intervalle de validité du tarif. Il ressort donc que le tarif $\mathrm{Y}_{2}$ est très précis dans le cubage des tiges de $R$. mangle. Quant à la régression $\mathrm{Y}_{3}$, l'imprécision est marquée par le fait que seules $17,6 \%$ des tiges ont leur volume dans ledit intervalle. Tenant compte des limites de validité des différentes régressions, le meilleur tarif $\left(\mathrm{Y}_{2}\right)$ a été représenté sous forme de table de cubage individuel (Tableau 4). Celle-ci permet de déterminer le volume d'une tige en croisant ses paramètres dendrométriques notamment la circonférence à une hauteur donnée $\left(\mathrm{C}_{0,1}\right)$ et sa longueur totale $(\mathrm{L})$.

\section{Tarifs de cubages de $R$. racemosa/harisonnii}

A la suite du prétraitement des données, 107 tiges de $R$. racemosa/harisonnii ont été échantillonnées pour élaborer des tarifs de cubage. Elles présentent l'échantillon homogène dont les caractéristiques dendrométriques sont consignées dans le Tableau 5. Cet échantillon de 107 tiges a permis d'élaborer trois régressions $\mathrm{Y}_{4}, \mathrm{Y}_{5}, \mathrm{Y}_{6}$ à l'aide du logiciel Statview5. Leurs coefficients de régression, paramètres statistiques d'appréciation des ajustements, sont consignés dans le Tableau 6. Ils ont permis d'établir les formules mathématiques de trois tarifs de cubage ainsi qu'il suit :

$\mathrm{Y}_{4}=0,001+0,037\left(\mathrm{C}_{0,1}\right)^{2} \mathrm{~L}$ avec $\mathrm{R}^{2}=0,956$;

$\mathrm{Y}_{5}=0,0001+0,059\left(\mathrm{C}_{0,1}\right)^{2}+0,029\left(\mathrm{C}_{0,1}\right)^{2} \mathrm{~L}$ avec

$\mathrm{R}^{2}=0,967$;

$\mathrm{Y}_{6}=0,00003+0,084\left(\mathrm{C}_{\mathrm{m}}\right)^{2} \mathrm{~L}$ avec $\mathrm{R} 2=0,989$. 
Analyse de la pertinence des tarifs de cubages de $R$. racemosa/harisonnii

Les paramètres statistiques considérés dans cette analyse sont les mêmes que ceux précédemment utilisés pour les tarifs de cubage de $R$. mangle (Tableau 7). Les valeurs des différents coefficients et la moyenne quadratique des résidus absolus et des résidus relatifs montrent que $\mathrm{Y}_{4}, \mathrm{Y}_{5}$ et $\mathrm{Y}_{6}$ sont des régressions pertinentes. Toutefois, la régression $\mathrm{Y}_{6}$ est la plus pertinente au regard des paramètres statistiques considérés. Elle donne théoriquement les meilleures estimations du volume des tiges de $R$. racemosa/harisonnii.

\section{Analyse de la précision des tarifs de cubage de $R$. racemosa/harisonnii}

Lorsque l'on analyse la précision des tarifs, en tenant compte de leurs limites de validité, il ressort que $\mathrm{Y}_{4}$ est la régression la plus précise pour $R$. racemosa/harisonnii. Cependant, seulement $19,62 \%$ des tiges ont leur volume dans l'intervalle : volume moyen \pm l'écart-type estimé des résidus. Ce pourcentage étant inférieur à $68 \%$ des tiges, $\mathrm{Y}_{4}$ bien que pertinent, par rapport aux paramètres statistiques étudiés, est considéré comme un tarif peu précis. Néanmoins, en considérant la nullité de la somme des résidus (volume mesuré-volume estimé $=0,06$ ), l'étude propose l'utilisation du tarif à l'aide d'une table de cubage individuel (Tableau 8).

Tableau 1 : Caractéristiques dendrométriques des tiges de Rhizophora mangle.

\begin{tabular}{lcccc}
\hline $\mathbf{L}(\mathbf{m})$ & $\mathbf{C}_{\mathbf{0}, \mathbf{1}}(\mathbf{m})$ & $\mathbf{C}_{\mathbf{m}}(\mathbf{m})$ & $\mathbf{C}_{\mathbf{f}}(\mathbf{m})$ & $\mathbf{Y}\left(\mathbf{m}^{\mathbf{3}}\right)$ \\
\hline 2,12 & 0,112 & 0,109 & 0,094 & 0,0019 \\
2,59 & 0,12 & 0,095 & 0,094 & 0,0020 \\
2,5 & 0,14 & 0,098 & 0,094 & 0,0022 \\
2,3 & 0,13 & 0,115 & 0,094 & 0,0024 \\
2,1 & 0,131 & 0,122 & 0,105 & 0,0024 \\
2,24 & 0,166 & 0,115 & 0,094 & 0,0027 \\
2,3 & 0,153 & 0,125 & 0,1 & 0,0029 \\
2,44 & 0,149 & 0,128 & 0,094 & 0,0031 \\
2,6 & 0,148 & 0,123 & 0,094 & 0,0031 \\
2,26 & 0,146 & 0,14 & 0,095 & 0,0033 \\
2,9 & 0,128 & 0,122 & 0,094 & 0,0033 \\
2,56 & 0,16 & 0,14 & 0,094 & 0,0038 \\
2,91 & 0,168 & 0,13 & 0,094 & 0,0040 \\
2 & 0,182 & 0,163 & 0,12 & 0,0041 \\
2,86 & 0,21 & 0,125 & 0,094 & 0,0044 \\
2,39 & 0,198 & 0,148 & 0,117 & 0,0045 \\
3 & 0,169 & 0,168 & 0,1 & 0,0060 \\
2,6 & 0,216 & 0,183 & 0,095 & 0,0065 \\
3,4 & 0,19 & 0,175 & 0,1 & 0,0076 \\
\hline
\end{tabular}


N. NDOUR et al. / Int. J. Biol. Chem. Sci. 8(5): 2155-2173, 2014

\begin{tabular}{|c|c|c|c|c|}
\hline 2,16 & 0,245 & 0,217 & 0,206 & 0,0083 \\
\hline 3 & 0,236 & 0,19 & 0,125 & 0,0086 \\
\hline 2,67 & 0,235 & 0,199 & 0,17 & 0,0086 \\
\hline 2,25 & 0,246 & 0,218 & 0,215 & 0,0089 \\
\hline 4,41 & 0,199 & 0,17 & 0,094 & 0,0096 \\
\hline 3,74 & 0,257 & 0,179 & 0,095 & 0,0101 \\
\hline 3,78 & 0,24 & 0,225 & 0,094 & 0,0135 \\
\hline 5,5 & 0,255 & 0,167 & 0,094 & 0,0135 \\
\hline 2,46 & 0,32 & 0,265 & 0,189 & 0,0137 \\
\hline 2,95 & 0,305 & 0,3 & 0,215 & 0,0195 \\
\hline 4,12 & 0,283 & 0,245 & 0,204 & 0,0198 \\
\hline 5,11 & 0,304 & 0,22 & 0,111 & 0,0202 \\
\hline 2 & 0,177 & 0,13 & 0,13 & 0,0031 \\
\hline 2,1 & 0,151 & 0,121 & 0,11 & 0,0026 \\
\hline 2,1 & 0,16 & 0,144 & 0,143 & 0,0036 \\
\hline 2,1 & 0,172 & 0,143 & 0,14 & 0,0036 \\
\hline 2,28 & 0,165 & 0,158 & 0,141 & 0,0044 \\
\hline 2,3 & 0,2 & 0,136 & 0,094 & 0,0037 \\
\hline 2,3 & 0,131 & 0,107 & 0,108 & 0,0023 \\
\hline 2,31 & 0,164 & 0,133 & 0,098 & 0,0033 \\
\hline 2,33 & 0,183 & 0,13 & 0,094 & 0,0034 \\
\hline 2,45 & 0,16 & 0,106 & 0,094 & 0,0026 \\
\hline 2,57 & 0,16 & 0,14 & 0,115 & 0,0040 \\
\hline 2,8 & 0,117 & 0,102 & 0,094 & 0,0024 \\
\hline 2,8 & 0,16 & 0,141 & 0,14 & 0,0046 \\
\hline 2,8 & 0,18 & 0,132 & 0,131 & 0,0044 \\
\hline 2,82 & 0,26 & 0,18 & 0,12 & 0,0079 \\
\hline 2,87 & 0,145 & 0,136 & 0,127 & 0,0042 \\
\hline 3 & 0,194 & 0,134 & 0,094 & 0,0047 \\
\hline 3,1 & 0,133 & 0,11 & 0,094 & 0,0031 \\
\hline 3,18 & 0,175 & 0,127 & 0,094 & 0,0044 \\
\hline 3,2 & 0,21 & 0,145 & 0,094 & 0,0058 \\
\hline 3,24 & 0,217 & 0,134 & 0,094 & 0,0055 \\
\hline 3,26 & 0,205 & 0,154 & 0,1 & 0,0064 \\
\hline 3,37 & 0,125 & 0,1 & 0,094 & 0,0029 \\
\hline 3,5 & 0,18 & 0,103 & 0,094 & 0,0039 \\
\hline 3,5 & 0,25 & 0,209 & 0,115 & 0,0116 \\
\hline 3,65 & 0,19 & 0,152 & 0,094 & 0,0067 \\
\hline
\end{tabular}

2161 
N. NDOUR et al. / Int. J. Biol. Chem. Sci. 8(5): 2155-2173, 2014

\begin{tabular}{|c|c|c|c|c|}
\hline 4 & 0,243 & 0,143 & 0,094 & 0,0079 \\
\hline 4,49 & 0,31 & 0,21 & 0,13 & 0,0172 \\
\hline 2,12 & 0,131 & 0,103 & 0,094 & 0,0019 \\
\hline 2,18 & 0,13 & 0,108 & 0,094 & 0,0021 \\
\hline 2,2 & 0,127 & 0,112 & 0,11 & 0,0023 \\
\hline 2,84 & 0,11 & 0,1 & 0,095 & 0,0023 \\
\hline 2,85 & 0,118 & 0,104 & 0,1 & 0,0025 \\
\hline 2,37 & 0,13 & 0,115 & 0,105 & 0,0025 \\
\hline 2,6 & 0,136 & 0,11 & 0,094 & 0,0026 \\
\hline 2,15 & 0,148 & 0,127 & 0,107 & 0,0028 \\
\hline 2,36 & 0,156 & 0,12 & 0,094 & 0,0028 \\
\hline 4,38 & 0,24 & 0,22 & 0,115 & 0,0154 \\
\hline 3,39 & 0,129 & 0,105 & 0,094 & 0,0031 \\
\hline 3 & 0,13 & 0,115 & 0,1 & 0,0032 \\
\hline 3,14 & 0,124 & 0,121 & 0,096 & 0,0035 \\
\hline 2,94 & 0,156 & 0,118 & 0,094 & 0,0035 \\
\hline 2,54 & 0,165 & 0,155 & 0,103 & 0,0045 \\
\hline 2,12 & 0,21 & 0,158 & 0,148 & 0,0047 \\
\hline 4,24 & 0,134 & 0,12 & 0,094 & 0,0048 \\
\hline 3,45 & 0,145 & 0,143 & 0,094 & 0,0051 \\
\hline 4,83 & 0,149 & 0,121 & 0,094 & 0,0057 \\
\hline 2,38 & 0,209 & 0,17 & 0,158 & 0,0058 \\
\hline 3,87 & 0,169 & 0,14 & 0,094 & 0,0059 \\
\hline 3 & 0,19 & 0,16 & 0,125 & 0,0061 \\
\hline 3,74 & 0,161 & 0,16 & 0,13 & 0,0072 \\
\hline 3,75 & 0,182 & 0,158 & 0,117 & 0,0073 \\
\hline 2,92 & 0,199 & 0,18 & 0,18 & 0,0078 \\
\hline 2,44 & 0,219 & 0,218 & 0,188 & 0,0089 \\
\hline 3,34 & 0,21 & 0,191 & 0,105 & 0,0089 \\
\hline 4,12 & 0,22 & 0,163 & 0,094 & 0,0089 \\
\hline 4,2 & 0,201 & 0,169 & 0,095 & 0,0091 \\
\hline 4,6 & 0,219 & 0,174 & 0,094 & 0,0109 \\
\hline 4,65 & 0,185 & 0,183 & 0,102 & 0,0110 \\
\hline 4,5 & 0,22 & 0,181 & 0,12 & 0,0116 \\
\hline 3,76 & 0,241 & 0,218 & 0,101 & 0,0129 \\
\hline 6 & 0,213 & 0,174 & 0,094 & 0,0140 \\
\hline 2,14 & 0,127 & 0,105 & 0,094 & 0,0020 \\
\hline 2,4 & 0,132 & 0,128 & 0,111 & 0,0030 \\
\hline
\end{tabular}




\begin{tabular}{|c|c|c|c|c|}
\hline 2,9 & 0,142 & 0,128 & 0,095 & 0,0036 \\
\hline 2,27 & 0,176 & 0,145 & 0,114 & 0,0039 \\
\hline 2,73 & 0,166 & 0,133 & 0,095 & 0,0039 \\
\hline 2,34 & 0,163 & 0,145 & 0,121 & 0,0039 \\
\hline 3 & 0,182 & 0,118 & 0,095 & 0,0039 \\
\hline 3 & 0,155 & 0,125 & 0,111 & 0,0039 \\
\hline 2,3 & 0,175 & 0,151 & 0,105 & 0,0041 \\
\hline 3,23 & 0,151 & 0,134 & 0,105 & 0,0045 \\
\hline 2,67 & 0,179 & 0,157 & 0,11 & 0,0051 \\
\hline 2,7 & 0,18 & 0,15 & 0,15 & 0,0052 \\
\hline 2,7 & 0,19 & 0,184 & 0,1 & 0,0065 \\
\hline 3 & 0,196 & 0,178 & 0,103 & 0,0070 \\
\hline 2,8 & 0,2 & 0,178 & 0,151 & 0,0070 \\
\hline 2,13 & 0,245 & 0,245 & 0,24 & 0,0101 \\
\hline 3 & 0,245 & 0,201 & 0,175 & 0,0100 \\
\hline 3,15 & 0,23 & 0,222 & 0,139 & 0,0113 \\
\hline 4,1 & 0,264 & 0,178 & 0,106 & 0,0113 \\
\hline 3,42 & 0,254 & 0,211 & 0,094 & 0,0114 \\
\hline 2,84 & 0,295 & 0,234 & 0,121 & 0,0121 \\
\hline 5,18 & 0,23 & 0,195 & 0,11 & 0,0149 \\
\hline 3,68 & 0,254 & 0,245 & 0,17 & 0,0163 \\
\hline 4,81 & 0,27 & 0,211 & 0,094 & 0,0166 \\
\hline 5,67 & 0,258 & 0,22 & 0,115 & 0,0206 \\
\hline 5,3 & 0,33 & 0,24 & 0,106 & 0,0247 \\
\hline 4,18 & 0,325 & 0,28 & 0,265 & 0,0271 \\
\hline 5,83 & 0,376 & 0,262 & 0,185 & 0,0348 \\
\hline 6,1 & 0,426 & 0,272 & 0,16 & 0,0407 \\
\hline 4,76 & 0,48 & 0,399 & 0,135 & 0,0559 \\
\hline 6,35 & 0,445 & 0,348 & 0,185 & 0,0604 \\
\hline 3,98 & 0,587 & 0,445 & 0,239 & 0,0630 \\
\hline
\end{tabular}

$\mathrm{Y}=$ volume en $\mathrm{m}^{3} ; \mathrm{C}_{0,1}=$ circonférence à $1 \mathrm{~cm}$ au-dessus des racines échasses $; \mathrm{C}_{\mathrm{f}}=$ circonférence au fin bout de la tige ; $\mathrm{C}_{\mathrm{m}}=$ circonférence au milieu de la tige ; $\mathrm{L}=$ longueur de la tige à cuber 
Tableau 2 : Coefficients de régression partielle des ajustements.

\begin{tabular}{lccc}
\hline Régressions & \multicolumn{3}{c}{ Coefficients de régression partielle } \\
\cline { 2 - 4 } & $\mathbf{a}_{\mathbf{0}}$ & $\mathbf{a}_{\mathbf{1}}$ & $\mathbf{a}_{\mathbf{2}}$ \\
\hline $\mathrm{Y}_{1}$ & 0,001 & 0,045 & - \\
$\mathrm{Y}_{2}$ & 0,0004 & 0,045 & 0,037 \\
$\mathrm{Y}_{3}$ & 0,0001 & 0,079 & - \\
\hline
\end{tabular}

Tableau 3: Paramètres statistiques des régressions.

\begin{tabular}{lccccc}
\hline Régressions & $\begin{array}{c}\text { Coefficients de } \\
\text { détermination }\end{array}$ & $\begin{array}{c}\text { Coefficients de } \\
\text { corrélation }\end{array}$ & $\begin{array}{c}\text { Coefficients de } \\
\text { variation résiduelle } \\
(\%)\end{array}$ & $\begin{array}{c}\text { Moyennes quadratiques } \\
\text { des résidus }\end{array}$ \\
\cline { 5 - 6 } & 0,965 & 0,982 & 22,25 & 0,0019 & 0,18 \\
$\mathrm{Y}_{1}$ & 0,968 & 0,984 & 21,27 & 0,0018 & 0,17 \\
$\mathrm{Y}_{2}$ & 0,993 & 0,997 & 9,72 & 0,0008 & 0,07 \\
$\mathrm{Y}_{3}$ & & & & \\
\hline
\end{tabular}

Tableau 4 : Coefficients de régression partiel des ajustements.

\begin{tabular}{lccc}
\hline Régressions & \multicolumn{3}{c}{ Coefficients de régression partielle } \\
\cline { 2 - 4 } & $\mathbf{a}_{\mathbf{0}}$ & $\mathbf{a}_{\mathbf{1}}$ & $\mathbf{a}_{\mathbf{2}}$ \\
\hline $\mathrm{Y}_{4}$ & 0,001 & 0,037 & - \\
$\mathrm{Y}_{5}$ & 0,0001 & 0,059 & 0,029 \\
$\mathrm{Y}_{6}$ & 0,00003 & 0,084 & - \\
\hline
\end{tabular}

Tableau 5: Paramètres statistiques des régressions.

\begin{tabular}{|c|c|c|c|c|c|}
\hline \multirow[t]{2}{*}{ Régressions } & \multirow[t]{2}{*}{$\begin{array}{l}\text { Coefficients de } \\
\text { détermination }\end{array}$} & \multirow[t]{2}{*}{$\begin{array}{l}\text { Coefficients de } \\
\text { corrélation }\end{array}$} & \multirow{2}{*}{$\begin{array}{c}\text { Coefficients de } \\
\text { variation résiduelle } \\
(\%)\end{array}$} & \multicolumn{2}{|c|}{$\begin{array}{c}\text { Moyennes quadratiques } \\
\text { des résidus }\end{array}$} \\
\hline & & & & absolu & relatifs \\
\hline $\mathrm{Y}_{4}$ & 0,956 & 0,978 & 23,4 & 0,003 & 0,17 \\
\hline $\mathrm{Y}_{5}$ & 0,967 & 0,983 & 20,8 & 0,003 & 0,17 \\
\hline$Y_{6}$ & 0,989 & 0,995 & 11,5 & 0,001 & 0,08 \\
\hline
\end{tabular}


Tableau 6 : Tarif de cubage de R. mangle.

\begin{tabular}{|c|c|c|c|c|c|c|c|c|c|c|c|c|c|c|}
\hline \multirow[t]{2}{*}{$\mathrm{C}_{0,1}(\mathrm{~m})$} & \multicolumn{14}{|c|}{ Longueur des tiges (m) } \\
\hline & 2 & 2,5 & 3 & 3,5 & 4 & 4,5 & 5 & 5,5 & 6 & 6,5 & 7 & 7,5 & 8 & 8,5 \\
\hline 0,11 & 0,0018 & 0,0021 & 0,0023 & 0,0025 & 0,0027 & 0,0030 & 0,0032 & 0,0034 & 0,0036 & 0,0039 & 0,0041 & 0,0043 & 0,0045 & 0,0047 \\
\hline 0,115 & 0,0020 & 0,0022 & 0,0025 & 0,0027 & 0,0030 &, 0032 & 0,0034 & 0,0037 & 0,0039 & 0,0042 & 0,0044 & 0,0047 & 0,0049 & 0,0052 \\
\hline 0,12 & 0,0021 & 0,0024 & 0,0026 & 0,0029 & 0,0032 & 0,0034 & 0,0037 & 0,0040 & 0,0042 & 0,0045 & 0,0048 & 0,0050 &, 0053 & 0,0056 \\
\hline 0,125 & 0,0023 & 0,0025 & 0,0028 & 0,0031 & 0,0034 & 0,0037 & 0,0040 & 0,0043 & 0,0046 & 0,0049 & 0,0052 & 0,0054 & 0,0057 & 0,0060 \\
\hline 0,13 & 0,0024 & 0,0027 & 0,0030 & 0,0033 & 0,0037 & 0,0040 & 0,0043 & 0,0046 & 0,0049 & 0,0052 & 0,0055 & & 0,0062 & 0,0065 \\
\hline 0,135 & 0026 & 0,0029 & 0,0032 & 0,0036 & 0,0039 & 0,0043 & 0,0046 & 0,0049 & 0,0053 & 0,0056 & 0,0059 & 0,0063 & 0,0066 & 0,0070 \\
\hline 0,14 & 0027 & 0,0031 & 0,0035 & 0,00 & 0,0042 & 0045 & 0,0049 & & 0,0056 & & & & & \\
\hline 0,145 & 0029 & 0,0033 & 0,0037 & 0,0041 & 0,0045 & 0,0048 & 0,0052 & 0,0056 & 0,0060 & 0,0064 & 0,0068 & 0,0072 & 0,0076 & 0,0080 \\
\hline 0,15 & 0031 & 0,0035 & 0,0039 & & & 0,0052 & & & 0,0064 & & & & & \\
\hline 0,155 & 0,0033 & 0,0037 & 0,0041 & 0,00 & 0,0050 & 0,0055 & 0,0059 & 0,0064 & 0,0068 & 0,0073 & 0,0077 & 0,0081 & 0,0086 & 0,0090 \\
\hline 0,16 & & 0,0039 & 0,0044 & & & 58 & & & 0,0072 & & & & & \\
\hline & & 0,00 & & 0,0 & 0,00 & & 0,00 & 0,0 & 0,00 & & 0,0 & 0,0 & 97 & 0,0102 \\
\hline 0,17 & 38 & 0,0044 & 0,0049 & 0,0054 & 0,0060 & 0,0065 & 0,0070 & 0,0 & 0,0081 & 0,0087 & 0,0 & 0,0 & 0,0103 & 0,0108 \\
\hline 0,175 & & & 0,0052 & & 0,0063 & 0,0069 & & & 0,0086 & & & & & 114 \\
\hline 0,18 & 43 & 0,0049 & 0,0055 & 0,00 & 0,0067 & 0,0073 & 0,0079 & 0,0 & 0,0091 & 0,0 & 0,0 & 0,0 & 0,0114 & 0,0120 \\
\hline 0,185 & 0045 & 0,0051 & 0,0057 & & 0,0070 & 0,0076 & 0,0083 & & 0,0095 & 0,0102 & 0,0 & 0,0114 & 121 & 0,0127 \\
\hline 0,19 & 0,0047 & 0,0054 & 0,0060 & 0,0067 & 0,0074 & 0,0080 & 0,0087 & 0,00 & 0,0100 & 0,0107 & 0,01 & 0,0120 & 0,0127 & 0,0134 \\
\hline 0,195 & 0,0049 & 0,0056 & 0,0063 & 0,0070 & 0,0077 & 0,0084 & 0,0091 & 0,0098 & 0,0106 & 0,0113 & 0,0120 & 0,0127 & 0,0134 & 0,0141 \\
\hline 0,2 & 0,0052 & 0,0059 & 0,0066 & 0,0074 & 0,0081 & 0,0089 & 0,0096 & & 0,0111 & 0,0118 & 0,0126 & & 0,0 & 0,0148 \\
\hline 0,205 & 0,0054 & 0,0062 & 0,0070 & 0,0077 & 0,0085 & 0,0093 & 0,0101 & 0,0108 & 0,0116 & 0,0124 & 0,0132 & 0,0140 & 0,0147 & 0,0155 \\
\hline 0,21 & & 0,0065 & 0,0073 & & & 0,0097 & & & 0,0122 & & 0,0138 & & & 0,0163 \\
\hline 0,215 & 0,0059 & 0,0068 & 0,0076 & 0,0085 & 0,0093 & 0,0102 & 0,0110 & 0,0119 & 0,0127 & 0,0136 & 0,0145 & 0,0153 & 0,0162 & 0,0170 \\
\hline 0,22 & 0,0062 & 0,0071 & 0,0080 & & & 0,0106 & & & & & & & 0,0169 & 0,0178 \\
\hline 0,225 & 0,0064 & 0,0074 & 0,0083 & 0,0092 & 0,0102 & 0,0111 & 0,0120 & 0,0130 & 0,0139 & 0,0149 & 0,0158 & 0,0167 & 0,0177 & 0,0186 \\
\hline 0,23 & 0,0067 & 0,0077 & 0,0087 & 0,0096 & 0,0106 & 0,0116 & 0,0126 & 0,0135 & 0,0145 & 0,0155 & 0,0165 & 0,0175 & 0,0184 & 0,0194 \\
\hline 0,235 & 0,0070 & 0,0080 & 0,0090 & 0,0100 & 0,0111 & 0,0121 & 0,0131 & 0,0141 & 0,0151 & 0,0162 & 0,0172 & 0,0182 & 0,0192 & 0,0203 \\
\hline 0,24 & 0,0073 & 0,0083 & 0,0094 & 0,0105 & 0,0115 & 0,0126 & 0,0136 & 0,0147 & 0,0158 & 0,0168 & 0,0179 & 0,0190 & 0,0200 & 0,0211 \\
\hline 0,245 & & 0,0087 & 0,0098 & & 0,0120 & 0,0131 & 0,0142 & & 0,0164 & 0,0175 & 0,0186 & 0,0198 & 0,0209 & 0,0220 \\
\hline 0,25 & 0,0078 & 0,0090 & 0,0102 & 0,0113 & 0,0125 & 0,0136 & 0,0148 & 0,0159 & 0,0171 & 0,0182 & 0,0194 & 0,0206 & 0,0217 & 0,0229 \\
\hline 0,255 & 0,0081 & 0,0093 & 0,0105 & 0,0117 & 0,0129 & 0,0142 & 0,0154 & 0,0166 & 0,0178 & 0,0190 & 0,0202 & 0,0214 & 0,0226 & 0,0238 \\
\hline 0,26 & 0,0084 & 0,0097 & 0,0109 & 0,0122 & 0,0134 & 0,0147 & 0,0159 & 0,0172 & 0,0184 & 0,0197 & 0,0210 & 0,0222 & 0,0235 & 0,0247 \\
\hline
\end{tabular}




\begin{tabular}{|c|c|c|c|c|c|c|c|c|c|c|c|c|c|c|}
\hline 0,265 & 0,0088 & 0,0101 & 0,0114 & 0,0127 & 0,0140 & 0,0153 & 0,0166 & 0,0179 & 0,0192 & 0,0204 & 0,0217 & 0,0230 & 0,0243 & 0,0256 \\
\hline 0,27 & 0,0091 & 0,0104 & 0,0118 & 0,0131 & 0,0145 & 0,0158 & 0,0172 & 0,0185 & 0,0199 & 0,0212 & 0,0226 & 0,0239 & 0,0253 & 0,0266 \\
\hline 0,275 & 0,0094 & 0,0108 & 0,0122 & 0,0136 & 0,0150 & 0,0164 & 0,0178 & 0,0192 & 0,0206 & 0,0220 & 0,0234 & 0,0248 & 0,0262 & 0,0276 \\
\hline 0,28 & 0,0097 & 0,0112 & 0,0126 & 0,0141 & 0,0155 & 0,0170 & 0,0184 & 0,0199 & 0,0213 & 0,0228 & 0,0242 & 0,0257 & 0,0271 & 0,0286 \\
\hline 0,285 & 0,0101 & 0,0116 & 0,0131 & 0,0146 & 0,0161 & 0,0176 & 0,0191 & 0,0206 & 0,0221 & 0,0236 & 0,0251 & 0,0266 & 0,0281 & 0,0296 \\
\hline 0,29 & 0,0104 & 0,0120 & 0,0135 & 0,0151 & 0,0166 & 0,0182 & 0,0197 & 0,0213 & 0,0229 & 0,0244 & 0,0260 & 0,0275 & 0,0291 & 0,0306 \\
\hline 0,295 & 0,0108 & 0,0124 & 0,0140 & 0,0156 & 0,0172 & 0,0188 & 0,0204 & 0,0220 & 0,0236 & 0,0252 & 0,0269 & 0,0285 & 0,0301 & 0,0317 \\
\hline 0,3 & 0,0111 & 0,0128 & 0,0144 & 0,0161 & 0,0178 & 0,0194 & 0,0211 & 0,0228 & 0,0244 & 0,0261 & 0,0278 & 0,0294 & 0,0311 & 0,0328 \\
\hline 0,305 & 0,0115 & 0,0132 & 0,0149 & 0,0166 & 0,0184 & 0,0201 & 0,0218 & 0,0235 & 0,0252 & 0,0270 & 0,0287 & 0,0304 & 0,0321 & 0,0338 \\
\hline 0,31 & 0,0118 & 0,0136 & 0,0154 & 0,0172 & 0,0189 & 0,0207 & 0,0225 & 0,0243 & 0,0261 & 0,0278 & 0,0296 & 0,0314 & 0,0332 & 0,0349 \\
\hline 0,315 & 0,0122 & 0,0140 & 0,0159 & 0,0177 & 0,0196 & 0,0214 & 0,0232 & 0,0251 & 0,0269 & 0,0287 & 0,0306 & 0,0324 & 0,0342 & 0,0361 \\
\hline 0,32 & 0,0126 & 0,0145 & 0,0164 & 0,0183 & 0,0202 & 0,0221 & 0,0240 & 0,0258 & 0,0277 & 0,0296 & 0,0315 & 0,0334 & 0,0353 & 0,0372 \\
\hline 0,325 & 0,0130 & 0,0149 & 0,0169 & 0,0188 & 0,0208 & 0,0227 & 0,0247 & 0,0266 & 0,0286 & 0,0306 & 0,0325 & 0,0345 & 0,0364 & 0,0384 \\
\hline 0,33 & 0,0134 & 0,0154 & 0,0174 & 0,0194 & 0,0214 & 0,0234 & 0,0254 & 0,0275 & 0,0295 & 0,0315 & 0,0335 & 0,0355 & 0,0375 & 0,0395 \\
\hline 0,335 & 0,0138 & 0,0158 & 0,0179 & 0,0200 & 0,0221 & 0,0241 & 0,0262 & 0,0283 & 0,0304 & 0,0324 & 0,0345 & 0,0366 & 0,0387 & 0,0407 \\
\hline 0,34 & 0,0142 & 0,0163 & 0,0184 & 0,0206 & 0,0227 & 0,0248 & 0,0270 & 0,0291 & 0,0313 & 0,0334 & 0,0355 & 0,0377 & 0,0398 & 0,0420 \\
\hline 0,345 & 0,0146 & 0,0168 & 0,0190 & 0,0212 & 0,0234 & 0,0256 & 0,0278 & 0,0300 & 0,0322 & 0,0344 & 0,0366 & 0,0388 & 0,0410 & 0,0432 \\
\hline 0,35 & 0,0150 & 0,0172 & 0,0195 & 0,0218 & 0,0240 & 0,0263 & 0,0286 & 0,0308 & 0,0331 & 0,0354 & 0,0376 & 0,0399 & 0,0422 & 0,0444 \\
\hline 0,355 & 0,0154 & 0,0177 & 0,0201 & 0,0224 & 0,0247 & 0,0271 & 0,0294 & 0,0317 & 0,0340 & 0,0364 & 0,0387 & 0,0410 & 0,0434 & 0,0457 \\
\hline 0,36 & 0,0158 & 0,0182 & 0,0206 & 0,0230 & 0,0254 & 0,0278 & 0,0302 & 0,0326 & 0,0350 & 0,0374 & 0,0398 & 0,0422 & 0,0446 & 0,0470 \\
\hline 0,365 & 0,0163 & 0,0187 & 0,0212 & 0,0236 & 0,0261 & 0,0286 & 0,0310 & 0,0335 & 0,0360 & 0,0384 & 0,0409 & 0,0434 & 0,0458 & 0,0483 \\
\hline 0,37 & 0,0167 & 0,0192 & 0,0218 & 0,0243 & 0,0268 & 0,0294 & 0,0319 & 0,0344 & 0,0370 & 0,0395 & 0,0420 & 0,0446 & 0,0471 & 0,0496 \\
\hline 0,375 & 0,0171 & 0,0197 & 0,0223 & 0,0249 & 0,0275 & 0,0301 & 0,0327 & 0,0353 & 0,0379 & 0,0405 & 0,0432 & 0,0458 & 0,0484 & 0,0510 \\
\hline 0,38 & 0,0176 & 0,0203 & 0,0229 & 0,0256 & 0,0283 & 0,0309 & 0,0336 & 0,0363 & 0,0390 & 0,0416 & 0,0443 & 0,0470 & 0,0496 & 0,0523 \\
\hline 0,385 & 0,0180 & 0,0208 & 0,0235 & 0,0263 & 0,0290 & 0,0317 & 0,0345 & 0,0372 & 0,0400 & 0,0427 & 0,0455 & 0,0482 & 0,0509 & 0,0537 \\
\hline 0,39 & 0,0185 & 0,0213 & 0,0241 & 0,0269 & 0,0298 & 0,0326 & 0,0354 & 0,0382 & 0,0410 & 0,0438 & 0,0466 & 0,0495 & 0,0523 & 0,0551 \\
\hline 0,395 & 0,0190 & 0,0219 & 0,0247 & 0,0276 & 0,0305 & 0,0334 & 0,0363 & 0,0392 & 0,0421 & 0,0449 & 0,0478 & 0,0507 & 0,0536 & 0,0565 \\
\hline 0,4 & 0,0194 & 0,0224 & 0,0254 & 0,0283 & 0,0313 & 0,0342 & 0,0372 & 0,0402 & 0,0431 & 0,0461 & 0,0490 & 0,0520 & 0,0550 & 0,0579 \\
\hline 0,405 & 0,0199 & 0,0230 & 0,0260 & 0,0290 & 0,0321 & 0,0351 & 0,0381 & 0,0412 & 0,0442 & 0,0472 & 0,0503 & 0,0533 & 0,0563 & 0,0594 \\
\hline 0,41 & 0,0204 & 0,0235 & 0,0266 & 0,0297 & 0,0328 & 0,0360 & 0,0391 & 0,0422 & 0,0453 & 0,0484 & 0,0515 & 0,0546 & 0,0577 & 0,0608 \\
\hline 0,415 & 0,0209 & 0,0241 & 0,0273 & 0,0305 & 0,0336 & 0,0368 & 0,0400 & 0,0432 & 0,0464 & 0,0496 & 0,0528 & 0,0559 & 0,0591 & 0,0623 \\
\hline 0,42 & 0,0214 & 0,0247 & 0,0279 & 0,0312 & 0,0344 & 0,0377 & 0,0410 & 0,0442 & 0,0475 & 0,0508 & 0,0540 & 0,0573 & 0,0606 & 0,0638 \\
\hline
\end{tabular}


Tableau 7: Caractéristiques dendrométriques des tiges de $R$. racemosa/harisonnii.

\begin{tabular}{|c|c|c|c|c|}
\hline $\mathbf{L}(\mathbf{m})$ & $\mathrm{C}_{0,1}(\mathrm{~m})$ & $C_{m}(m)$ & $\mathrm{C}_{\mathrm{f}}(\mathrm{m})$ & $Y\left(\mathbf{m}^{3}\right)$ \\
\hline 2 & 0,141 & 0,115 & 0,0942 & 0,0022 \\
\hline 2,5 & 0,172 & 0,122 & 0,095 & 0,0033 \\
\hline 2,87 & 0,187 & 0,11 & 0,096 & 0,0035 \\
\hline 2,8 & 0,17 & 0,123 & 0,098 & 0,0037 \\
\hline 3 & 0,19 & 0,115 & 0,095 & 0,0039 \\
\hline 2,72 & 0,181 & 0,133 & 0,095 & 0,0041 \\
\hline 3,17 & 0,179 & 0,124 & 0,095 & 0,0043 \\
\hline 3,46 & 0,178 & 0,126 & 0,0942 & 0,0048 \\
\hline 2,6 & 0,191 & 0,153 & 0,131 & 0,0051 \\
\hline 2,75 & 0,221 & 0,145 & 0,135 & 0,0055 \\
\hline 2,74 & 0,175 & 0,165 & 0,141 & 0,0058 \\
\hline 3,6 & 0,213 & 0,132 & 0,1 & 0,006 \\
\hline 4,7 & 0,167 & 0,13 & 0,0942 & 0,0065 \\
\hline 3,54 & 0,228 & 0,147 & 0,101 & 0,007 \\
\hline 2,35 & 0,248 & 0,195 & 0,159 & 0,0074 \\
\hline 3,75 & 0,201 & 0,162 & 0,141 & 0,0082 \\
\hline 3,79 & 0,22 & 0,165 & 0,095 & 0,0084 \\
\hline 3,74 & 0,241 & 0,166 & 0,0942 & 0,0088 \\
\hline 5,7 & 0,218 & 0,153 & 0,0942 & 0,0113 \\
\hline 4,6 & 0,296 & 0,166 & 0,0942 & 0,0126 \\
\hline 3,38 & 0,3 & 0,232 & 0,118 & 0,0143 \\
\hline 6,7 & 0,233 & 0,205 & 0,1 & 0,0207 \\
\hline 6,34 & 0,302 & 0,206 & 0,11 & 0,023 \\
\hline 3,23 & 0,372 & 0,297 & 0,221 & 0,0231 \\
\hline 6,55 & 0,306 & 0,211 & 0,00942 & 0,0236 \\
\hline 4,5 & 0,371 & 0,26 & 0,102 & 0,025 \\
\hline 7,5 & 0,34 & 0,193 & 0,095 & 0,0272 \\
\hline 4,18 & 0,375 & 0,291 & 0,205 & 0,0289 \\
\hline 8,56 & 0,35 & 0,196 & 0,0942 & 0,0324 \\
\hline 4,91 & 0,572 & 0,365 & 0,228 & 0,0594 \\
\hline 2,33 & 0,119 & 0,1 & 0,0942 & 0,0019 \\
\hline 2,12 & 0,148 & 0,107 & 0,0942 & 0,0022 \\
\hline 2,5 & 0,124 & 0,108 & 0,0942 & 0,0024 \\
\hline 2,18 & 0,149 & 0,113 & 0,0942 & 0,0024 \\
\hline 2,75 & 0,143 & 0,1 & 0,0942 & 0,0025 \\
\hline 2,89 & 0,136 & 0,109 & 0,0942 & 0,0029 \\
\hline
\end{tabular}




\begin{tabular}{|c|c|c|c|c|}
\hline 2,5 & 0,18 & 0,118 & 0,095 & 0,0032 \\
\hline 2,39 & 0,17 & 0,13 & 0,0942 & 0,0033 \\
\hline 2,34 & 0,149 & 0,136 & 0,113 & 0,0034 \\
\hline 2,28 & 0,188 & 0,144 & 0,0942 & 0,0038 \\
\hline 2,65 & 0,203 & 0,122 & 0,0942 & 0,0039 \\
\hline 2,13 & 0,195 & 0,151 & 0,0942 & 0,0039 \\
\hline 2,87 & 0,17 & 0,132 & 0,0942 & 0,0041 \\
\hline 3,3 & 0,157 & 0,13 & 0,115 & 0,0046 \\
\hline 3,4 & 0,176 & 0,136 & 0,0942 & 0,0051 \\
\hline 3,32 & 0,205 & 0,129 & 0,0942 & 0,0052 \\
\hline 2,21 & 0,199 & 0,175 & 0,16 & 0,0055 \\
\hline 3,9 & 0,176 & 0,132 & 0,0942 & 0,0057 \\
\hline 3,19 & 0,196 & 0,15 & 0,113 & 0,006 \\
\hline 2,8 & 0,211 & 0,166 & 0,102 & 0,0061 \\
\hline 4,65 & 0,175 & 0,129 & 0,0942 & 0,0065 \\
\hline 2,26 & 0,225 & 0,202 & 0,16 & 0,0072 \\
\hline 4,56 & 0,172 & 0,146 & 0,0942 & 0,0075 \\
\hline 3,45 & 0,207 & 0,167 & 0,1 & 0,0075 \\
\hline 4,25 & 0,215 & 0,141 & 0,0942 & 0,0076 \\
\hline 4,8 & 0,188 & 0,147 & 0,0942 & 0,0083 \\
\hline 4,63 & 0,219 & 0,151 & 0,0942 & 0,0091 \\
\hline 4,4 & 0,238 & 0,165 & 0,11 & 0,0104 \\
\hline 4,5 & 0,234 & 0,166 & 0,0942 & 0,0104 \\
\hline 3,75 & 0,234 & 0,189 & 0,128 & 0,0107 \\
\hline 5,8 & 0,206 & 0,16 & 0,0942 & 0,0118 \\
\hline 5 & 0,24 & 0,178 & 0,102 & 0,0129 \\
\hline 4,46 & 0,274 & 0,184 & 0,0942 & 0,013 \\
\hline 2,87 & 0,37 & 0,273 & 0,218 & 0,0184 \\
\hline 6,5 & 0,3 & 0,24 & 0,095 & 0,0284 \\
\hline 6,2 & 0,353 & 0,233 & 0,101 & 0,029 \\
\hline 5,72 & 0,352 & 0,28 & 0,098 & 0,0339 \\
\hline 7,52 & 0,337 & 0,249 & 0,112 & 0,0373 \\
\hline 2,9 & 0,503 & 0,45 & 0,32 & 0,0448 \\
\hline 8,32 & 0,517 & 0,354 & 0,139 & 0,087 \\
\hline 2,47 & 0,196 & 0,151 & 0,115 & 0,0047 \\
\hline 2,47 & 0,189 & 0,142 & 0,0942 & 0,0041 \\
\hline 2,58 & 0,16 & 0,155 & 0,096 & 0,0045 \\
\hline 2,83 & 0,235 & 0,218 & 0,118 & 0,0097 \\
\hline 3,52 & 0,177 & 0,133 & 0,098 & 0,0052 \\
\hline 3,67 & 0,188 & 0,14 & 0,101 & 0,006 \\
\hline
\end{tabular}




\begin{tabular}{|c|c|c|c|c|}
\hline 3,74 & 0,2 & 0,146 & 0,104 & 0,0068 \\
\hline 2 & 0,141 & 0,115 & 0,0942 & 0,0022 \\
\hline 2,5 & 0,172 & 0,122 & 0,095 & 0,0033 \\
\hline 2,87 & 0,187 & 0,11 & 0,096 & 0,0035 \\
\hline 2,8 & 0,17 & 0,123 & 0,098 & 0,0037 \\
\hline 3 & 0,19 & 0,115 & 0,095 & 0,0039 \\
\hline 2,72 & 0,181 & 0,133 & 0,095 & 0,0041 \\
\hline 3,17 & 0,179 & 0,124 & 0,095 & 0,0043 \\
\hline 3,46 & 0,178 & 0,126 & 0,0942 & 0,0048 \\
\hline 2,6 & 0,191 & 0,153 & 0,131 & 0,0051 \\
\hline 2,75 & 0,221 & 0,145 & 0,135 & 0,0055 \\
\hline 2,74 & 0,175 & 0,165 & 0,141 & 0,0058 \\
\hline 3,6 & 0,213 & 0,132 & 0,1 & 0,006 \\
\hline 4,7 & 0,167 & 0,13 & 0,0942 & 0,0065 \\
\hline 3,54 & 0,228 & 0,147 & 0,101 & 0,007 \\
\hline 2,35 & 0,248 & 0,195 & 0,159 & 0,0074 \\
\hline 3,75 & 0,201 & 0,162 & 0,141 & 0,0082 \\
\hline 3,79 & 0,22 & 0,165 & 0,095 & 0,0084 \\
\hline 3,74 & 0,241 & 0,166 & 0,0942 & 0,0088 \\
\hline 5,7 & 0,218 & 0,153 & 0,0942 & 0,0113 \\
\hline 4,6 & 0,296 & 0,166 & 0,0942 & 0,0126 \\
\hline 3,38 & 0,3 & 0,232 & 0,118 & 0,0143 \\
\hline 6,7 & 0,233 & 0,205 & 0,1 & 0,0207 \\
\hline 6,34 & 0,302 & 0,206 & 0,11 & 0,023 \\
\hline 3,23 & 0,372 & 0,297 & 0,221 & 0,0231 \\
\hline 6,55 & 0,306 & 0,211 & 0,00942 & 0,0236 \\
\hline 4,5 & 0,371 & 0,26 & 0,102 & 0,025 \\
\hline 7,5 & 0,34 & 0,193 & 0,095 & 0,0272 \\
\hline 4,18 & 0,375 & 0,291 & 0,205 & 0,0289 \\
\hline 8,56 & 0,35 & 0,196 & 0,0942 & 0,0324 \\
\hline 4,91 & 0,572 & 0,365 & 0,228 & 0,0594 \\
\hline
\end{tabular}


Tableau 8 : Tarif de cubage de $R$. racemosa/harisonnii.

\begin{tabular}{|c|c|c|c|c|c|c|c|c|c|c|c|c|c|c|}
\hline \multirow[t]{2}{*}{$\mathrm{C}_{0,1}(\mathrm{~m})$} & \multicolumn{14}{|c|}{ Longueur des tiges (m) } \\
\hline & 2 & 2,5 & 3 & 3,5 & 4 & 4,5 & 5 & 5,5 & 6 & 6,5 & 7 & 7,5 & 8 & 8,5 \\
\hline 0,15 & 0,0027 & 0,0031 & 0,0035 & 0,0039 & 0,0043 & 0,0047 & 0,0052 & 0,0056 & 0,0060 & 0,0064 & 0,0127 & 0,0072 & 0,0077 & 0,0081 \\
\hline 0,155 & 0,0028 & 0,0032 & 0,0037 & 0,0041 & 0,0046 & 0,0050 & 0,0054 & 0,0059 & 0,0063 & 0,0068 & 0,0132 & 0,0077 & 0,0081 & 0,0086 \\
\hline 0,16 & 0,0029 & 0,0034 & 0,0038 & 0,0043 & 0,0048 & 0,0053 & 0,0057 & 0,0062 & 0,0067 & 0,0072 & 0,0138 & 0,0081 & 0,0086 & 0,0091 \\
\hline 0,165 & 0,0030 & 0,0035 & 0,0040 & 0,0045 & 0,0050 & 0,0055 & 0,0060 & 0,0065 & 0,0070 & 0,0075 & 0,0145 & 0,0086 & 0,0091 & 0,0096 \\
\hline 0,17 & 0,0031 & 0,0037 & 0,0042 & 0,0047 & 0,0053 & 0,0058 & 0,0063 & 0,0069 & 0,0074 & 0,0080 & 0,0151 & 0,0090 & 0,0096 & 0,0101 \\
\hline 0,175 & 0,0033 & 0,0038 & 0,0044 & 0,0050 & 0,0055 & 0,0061 & 0,0067 & 0,0072 & 0,0078 & 0,0084 & 0,0157 & 0,0095 & 0,0101 & 0,0106 \\
\hline 0,18 & 0,0034 & 0,0040 & 0,0046 & 0,0052 & 0,0058 & 0,0064 & 0,0070 & 0,0076 & 0,0082 & 0,0088 & 0,0164 & 0,0100 & 0,0106 & 0,0112 \\
\hline 0,185 & 0,0035 & 0,0042 & 0,0048 & 0,0054 & 0,0061 & 0,0067 & 0,0073 & 0,0080 & 0,0086 & 0,0092 & 0,0171 & 0,0105 & 0,0111 & 0,0118 \\
\hline 0,19 & 0,0037 & 0,0043 & 0,0050 & 0,0057 & 0,0063 & 0,0070 & 0,0077 & 0,0083 & 0,0090 & 0,0097 & 0,0177 & 0,0110 & 0,0117 & 0,0124 \\
\hline 0,195 & 0,0038 & 0,0045 & 0,0052 & 0,0059 & 0,0066 & 0,0073 & 0,0080 & 0,0087 & 0,0094 & 0,0101 & 0,0184 & 0,0116 & 0,0123 & 0,0130 \\
\hline 0,2 & 0,0040 & 0,0047 & 0,0054 & 0,0062 & 0,0069 & 0,0077 & 0,0084 & 0,0091 & 0,0099 & 0,0106 & 0,0191 & 0,0121 & 0,0128 & 0,0136 \\
\hline 0,205 & 0,0041 & 0,0049 & 0,0057 & 0,0064 & 0,0072 & 0,0080 & 0,0088 & 0,0096 & 0,0103 & 0,0111 & 0,0198 & 0,0127 & 0,0134 & 0,0142 \\
\hline 0,21 & 0,0043 & 0,0051 & 0,0059 & 0,0067 & 0,0075 & 0,0083 & 0,0092 & 0,0100 & 0,0108 & 0,0116 & 0,0206 & 0,0132 & 0,0141 & 0,0149 \\
\hline 0,215 & 0,0044 & 0,0053 & 0,0061 & 0,0070 & 0,0078 & 0,0087 & 0,0096 & 0,0104 & 0,0113 & 0,0121 & 0,0213 & 0,0138 & 0,0147 & 0,0155 \\
\hline 0,22 & 0,0046 & 0,0055 & 0,0064 & 0,0073 & 0,0082 & 0,0091 & 0,0100 & 0,0108 & 0,0117 & 0,0126 & 0,0221 & 0,0144 & 0,0153 & 0,0162 \\
\hline 0,225 & 0,0047 & 0,0057 & 0,0066 & 0,0076 & 0,0085 & 0,0094 & 0,0104 & 0,0113 & 0,0122 & 0,0132 & 0,0229 & 0,0150 & 0,0160 & 0,0169 \\
\hline 0,23 & 0,0049 & 0,0059 & 0,0069 & 0,0079 & 0,0088 & 0,0098 & 0,0108 & 0,0118 & 0,0127 & 0,0137 & 0,0236 & 0,0157 & 0,0167 & 0,0176 \\
\hline 0,235 & 0,0051 & 0,0061 & 0,0071 & 0,0082 & 0,0092 & 0,0102 & 0,0112 & 0,0122 & 0,0133 & 0,0143 & 0,0244 & 0,0163 & 0,0173 & 0,0184 \\
\hline 0,24 & 0,0053 & 0,0063 & 0,0074 & 0,0085 & 0,0095 & 0,0106 & 0,0117 & 0,0127 & 0,0138 & 0,0149 & 0,0252 & 0,0170 & 0,0180 & 0,0191 \\
\hline 0,245 & 0,0054 & 0,0066 & 0,0077 & 0,0088 & 0,0099 & 0,0110 & 0,0121 & 0,0132 & 0,0143 & 0,0154 & 0,0261 & 0,0177 & 0,0188 & 0,0199 \\
\hline 0,25 & 0,0056 & 0,0068 & 0,0079 & 0,0091 & 0,0103 & 0,0114 & 0,0126 & 0,0137 & 0,0149 & 0,0160 & 0,0269 & 0,0183 & 0,0195 & 0,0207 \\
\hline 0,255 & 0,0058 & 0,0070 & 0,0082 & 0,0094 & 0,0106 & 0,0118 & 0,0130 & 0,0142 & 0,0154 & 0,0166 & 0,0277 & 0,0190 & 0,0202 & 0,0215 \\
\hline 0,26 & 0,0060 & 0,0073 & 0,0085 & 0,0098 & 0,0110 & 0,0123 & 0,0135 & 0,0148 & 0,0160 & 0,0173 & 0,0286 & 0,0198 & 0,0210 & 0,0223 \\
\hline 0,265 & 0,0062 & 0,0075 & 0,0088 & 0,0101 & 0,0114 & 0,0127 & 0,0140 & 0,0153 & 0,0166 & 0,0179 & 0,0295 & 0,0205 & 0,0218 & 0,0231 \\
\hline 0,27 & 0,0064 & 0,0077 & 0,0091 & 0,0104 & 0,0118 & 0,0131 & 0,0145 & 0,0158 & 0,0172 & 0,0185 & 0,0304 & 0,0212 & 0,0226 & 0,0239 \\
\hline 0,275 & 0,0066 & 0,0080 & 0,0094 & 0,0108 & 0,0122 & 0,0136 & 0,0150 & 0,0164 & 0,0178 & 0,0192 & 0,0313 & 0,0220 & 0,0234 & 0,0248 \\
\hline 0,28 & 0,0068 & 0,0083 & 0,0097 & 0,0112 & 0,0126 & 0,0141 & 0,0155 & 0,0170 & 0,0184 & 0,0199 & 0,0322 & 0,0228 & 0,0242 & 0,0257 \\
\hline 0,285 & 0,0070 & 0,0085 & 0,0100 & 0,0115 & 0,0130 & 0,0145 & 0,0160 & 0,0175 & 0,0190 & 0,0205 & 0,0331 & 0,0235 & 0,0250 & 0,0265 \\
\hline 0,29 & 0,0072 & 0,0088 & 0,0103 & 0,0119 & 0,0134 & 0,0150 & 0,0166 & 0,0181 & 0,0197 & 0,0212 & 0,0340 & 0,0243 & 0,0259 & 0,0274 \\
\hline 0,295 & 0,0074 & 0,0090 & 0,0107 & 0,0123 & 0,0139 & 0,0155 & 0,0171 & 0,0187 & 0,0203 & 0,0219 & 0,0350 & 0,0251 & 0,0268 & 0,0284 \\
\hline 0,3 & 0,0077 & 0,0093 & 0,0110 & 0,0127 & 0,0143 & 0,0160 & 0,0177 & 0,0193 & 0,0210 & 0,0226 & 0,0360 & 0,0260 & 0,0276 & 0,0293 \\
\hline 0,42 & 0,0141 & 0,0173 & 0,0206 & 0,0238 & 0,0271 & 0,0304 & 0,0336 & 0,0369 & 0,0402 & 0,0434 & 0,0630 & 0,0500 & 0,0532 & 0,0565 \\
\hline
\end{tabular}




\section{DISCUSSION}

\section{Estimateur de la biomasse volumique des tiges de mangrove}

La sélection des tiges destinées à l'élaboration des tarifs de cubages a été faite dans l'intervalle (coefficient moyen \pm 2 fois l'écart type). Cette méthode a permis d'éliminer les tiges de forme irrégulière et de sélectionner celles qui se rapprochent le plus des quatre solides géométriques (Cylindre, Paraboloïde, Cône et Néloïde) pour leur cubage à l'aide de la formule de Newton. Le choix des ajustements de type linéaire est lié au fait que les équations transformés sont sources de biais liés à une sous-estimation du volume des tiges selon Rondeux (1993). De plus, les ajustements linéaires choisis dans le cadre de cette étude ont déjà été testés avec succès dans la mangrove de Sangareya en république de Guinée par Arsenault (1993).

\section{Analyse comparative des tarifs de cubages}

Les résultats obtenus montrent que pour $R$. mangle aussi bien que pour $R$. racemosa/harisonnii, les tarifs de cubage sont pertinents lorsque l'analyse de leur performance se limite aux coefficients de détermination, de corrélation, de variation résiduelle et aux moyennes quadratique des résidus absolus et des résidus relatifs. Eu égard à ces paramètres, le test uni-varié montre qu'il n'y a pas de différence significative entre les régressions élaborées $(\mathrm{P}=0.3)$. Cependant, lorsque que l'on considère les limites de validité des tarifs définies par l'intervalle: volume moyen \pm l'écart-type estimé des résidus, seul le tarif $\mathrm{Y}_{2}$ révèle une bonne précision pour $R$. mangle. C'est pour cette principale raison que l'étude retient $\mathrm{Y}_{2}=$ $0,0004+0,045\left(\mathrm{C}_{0,1}\right)^{2}+0,037\left(\mathrm{C}_{0,1}\right)^{2} \mathrm{~L}$ avec $\mathrm{R}^{2}=$ 0,968 comme le meilleur tarif de cubage pour $R$. mangle au Delta du Saloum. Concernant $R$. racemosa/harisonnii, $\mathrm{Y}_{4}$ est la régression la plus pertinente avec des limites de validité assez étroites. En fait, cette faible précision du tarif est liée au fait que seulement $19,62 \%$ des tiges ont leur volume dans ses limites de validité. Il serait aussi possible de se fonder sur l'élargissement de l'intervalle de validité qui deviendra : volume moyen \pm 2 fois la valeur de l'écart-type estimé des résidus. A cette condition, 36,44\% des tiges auront leur volume dans l'intervalle de validité du tarif, ce qui améliorerait relativement la précision du tarif.

Par ailleurs, les modèles d'ajustements élaborés dans le cadre de cette étude sont plus pertinents que ceux élaborés par Arsenault (1993) pour la mangrove de Sangareya en Guinée Conakry. En fait, Arsenault a testé le même ajustement : $\mathrm{Y}=\mathrm{a}_{\mathrm{o}}+\mathrm{a}_{1} \mathrm{C}_{\mathrm{b}}{ }^{2} \mathrm{~L}$ avec un échantillon composite de 192 tiges (95 Rhizophora mangle et 97 Avicennia germinans). Ayant obtenu une régression avec un $\mathrm{R}^{2}=0,63$, l'on constate que sa régression est moins pertinente que les nôtres. Cette différence de pertinence des régressions pourrait être liée à la nature de l'échantillon qui est issu de peuplements quasi mono spécifiques au Delta du Saloum et d'un mélange de tiges d'espèces différentes à Sangareya. Au Sénégal, Doyen et al. (1985) ont travaillé dans l'unité (U4) bien avant notre étude et élaboré cinq tarifs de cubage à partir de modèles soient logarithmiques ou exponentiels. Les coefficients de corrélation de leurs tarifs de cubage ont varié entre 0,993 et 0,997 alors que leurs coefficients de variation résiduelle ont varié entre $13,6 \%$ et $22,4 \%$. Ces différents paramètres statistiques montrent que leurs régressions sont quasiment aussi pertinentes que les nôtres. Cependant, les statistiques générés par les modèles (logarithmes et exponentiels) pour l'estimation du volume des tiges font l'objet de débat entre les spécialistes de la dendrométrie. En fait, lors d'une transformation logarithmique, la droite résultante de l'équation ne passe plus par la moyenne des valeurs du volume des tiges, mais plutôt par la moyenne des logarithmes de ces valeurs. Les fondamentalistes pensent que la fonction logarithme (équation transformée) génère un coefficient de variation résiduel qui traduit l'erreur susceptible d'être commise sur la valeur moyenne de la variable dépendante. Mieux encore, même après le retour aux variables initiales, l'ajustement passera par la moyenne géométrique des volumes au lieu de leur moyenne arithmétique (Rondeux, 1993). L'effet de la transformation consiste donc à sous-estimer systématiquement le volume des tiges. D'ailleurs, d'autres auteurs soutiennent que la transformation logarithmique conduit souvent à un modèle non linéaire (Návar et al., 2002; SaintAndré et al., 2005). Ces différentes considérations nous amènent à recommander l'utilisation d'ajustements non transformés pour la mangrove du Delta du Saloum.

\section{Applicabilité des tarifs élaborés}

Sur la base des résultats obtenus, $\mathrm{Y}_{2}$ est applicable pour $R$. mangle au Delta du Saloum. Toutefois, les circonférences à la base devraient être comprises dans l'intervalle ]0,10 - 0,43 m[. Cette précaution s'impose lorsque l'on doit respecter les limites de validités du tarif selon la théorie de Rondeux. D'ailleurs, il est à constater que les calibres du bois exploité par les populations se situent dans cet intervalle. Concernant $R$. racemosa/harisonnii, $\mathrm{Y}_{4}$ serait applicable avec des 
tiges de circonférences comprises entre ]0,15 - 0,43 $\mathrm{m}]$. Il apparait donc que les intervalles de validité de ces deux tarifs conduisent quasiment aux mêmes mesures de circonférence pour le genre Rhizophora. Bien que $\mathrm{Y}_{4}$ soit de faible précision, l'examen de la nullité des résidus entre le volume mesuré et celui estimé, montre que leur somme est de 0,06. Cette faible valeur $(>0)$ indique une sous-estimation $\mathrm{du}$ volume des tiges par $\mathrm{Y}_{4}$. Sur la base de ce critère d'analyse et dans l'hypothèse de mesures de circonférence en quantité proportionnelle à droite et à gauche des limites de validité du tarif, l'utilisation de l'ajustement serait sans risque de biaiser considérablement les mesures de volume des tiges de $R$. racemosa/harisonnii. Pour ces diverses raisons, l'étude recommande aussi l'utilisation des régressions $\mathrm{Y}_{2}$ et $\mathrm{Y}_{4}$ au Delta du Saloum.

\section{Conclusion}

Le présent article indique l'existence d'un besoin en bois de palétuvier des populations du Delta au moment où la ressource a amorcé sa dégradation. Il montre la nécessité de mettre en place des mécanismes de gestion durable de la mangrove du Delta du Saloum. Il a permis d'élaborer six régressions $\left(\mathrm{Y}_{1}, \mathrm{Y}_{2}, \mathrm{Y}_{3}, \mathrm{Y}_{4}, \mathrm{Y}_{5}\right.$, et $\left.\mathrm{Y}_{6}\right)$ dont la pertinence varie en fonction de l'espèce de palétuvier et des paramètres dendrométriques étudiés. En termes de validité, le tarif de type $\mathrm{Y}_{2}=$ $0,0004+0,045\left(\mathrm{C}_{0}\right)^{2}+0,037\left(\mathrm{C}_{0,1}\right)^{2} \mathrm{~L}$ a été le plus précis pour $R$. mangle alors que $\mathrm{Y}_{4}=0,001+0,037$ $\left(\mathrm{C}_{0,1}\right)^{2} \mathrm{~L}$ a été le plus précis pour $R$. racemosa/harisonnii. Ainsi, ces deux tarifs $\left(\mathrm{Y}_{2}\right.$ et $\mathrm{Y}_{4}$ ) pourraient être utilisés dans l'estimation de la biomasse volumique des forêts de mangrove du Delta du Saloum en tenant compte, bien sûr, du degré de précision souhaitée.

\section{REMERCIEMENTS}

L'Institut des Sciences de l'Environnement de l'Université Cheikh Anta Diop de Dakar remercie vivement la Faculté des Sciences Agronomiques de Gembloux (FUSAGx) et la Direction des Parcs Nationaux (DPN) du Sénégal pour leur appui financier, logistique et institutionnel au cours du présent travail de recherche. Les auteurs dédient une mention spéciale à Monsieur Hervé Pirard, coordonnateur du projet mangrove et à Monsieur Souleymane Mansaly, agent des parcs nationaux et coordonnateur national du projet.

\section{REFERENCES}

Arsenault D. 1993. Résultats et analyse de l'inventaire forestier et autres études dendrométriques. Rapport de projet, document n $11 / 92$, République de Guinée.

Dallmeier F. 1992. Long-term monitoring of biological diversity tropical forest Areas. In Tropical Forest Methods for Establishment and Inventory of Permanent Plot. MAB digest, Unesco: Paris; 1-72.

Dia IMM. 2003. Elaboration et Mise en Euvre du Plan de Gestion Intégré, la Réserve de Biosphère du Delta du Saloum, Sénégal. UICN, Gland : Suisse, Cambridge et RoyaumeUni.

Dieye EHB, Diaw AT, Sané T, Ndour N. 2013. Dynamique de la mangrove de l'estuaire du Saloum (Sénégal) entre1972 et 2010 dans la rubrique Environnement, Nature, Paysage. Revue Cybergeo, 629: 1-22.

Dione D, Sy AB, Ndiaye MS. 2005. Contribution économique et sociale de la pêche artisanale au Sénégal dans le cadre du programme pour des Moyens d'Existence Durables dans la Pêche en Afrique de l'Ouest, projet pilote 1 «Moyens d'existence améliorés dans le secteur Postcapture de la pêche artisanale » au Cameroun, en Gambie, au Sénégal et au Tchad.

Doyen A, Agboba C. 1985. La Mangrove à Usages Multiples de l'Estuaire du Saloum (Sénégal). Unesco-MAB-COMAR: Paris.

Faye MN, Kaly JL, Diallo A, Guissé A. 2008. Influence des facteurs édaphiques sur la biomasse foliaire de deux espèces de palétuviers (Rhizophora sp., Avicennia africana) dans la Réserve de Biosphère du Delta du Saloum, Sénégal. Journal des Sciences et Technologies, 7: 35-42.

Leruse G. 2000. Estimation de la consommation de bois de mangrove pour les populations de la RBDS (Sénégal) et proposition de méthode de gestion, mémoire de fin d'études. Dakar, Sénégal.

Návar J, Méndez E, Dale V. 2002. Estimating stand biomass in the Tamaulipan thornscrub of northeastern Mexico. Annals of Forest Science, 59(8): 813-821.

Ndiongue B. 2003. Etudes des impacts socioéconomiques et écologiques du fumage de poissons dans la réserve de Biosphère du Delta du Saloum. Rapport de projet mangrove.

Ndour N. 2005. Caractérisation et étude de la dynamique des peuplements de mangrove du Delta du Saloum (République du Sénégal). Thèse de troisième cycle, Dakar, Faculté des Sciences et techniques, Université Cheikh Anta Diop de Dakar, p180. 
Ndour N, Sambou B, Dieng SD. 2013. Production de litière de la mangrove du Parc National du Delta du Saloum (Sénégal). Int. J. Biol. Chem. Sci., 7(6): 2349-2369.

Picard N, Laurent SA, Henry M. 2012. Manuel de Construction d'Equations Allométriques pour l'Estimation du Volume et la Biomasse des Arbres- de la Mesure de Terrain à la Prédiction. CIRAD.

Pirard H, L'hoir V. 2002. Profession : coupeur de perches. Etude de l'exploitation et de la commercialisation des perches de palétuviers (Rhizophora racemosa/harionnii) dans la Réserve de Biosphère du Delta du Saloum (Sénégal).Dakar, Sénégal.
Rondeux J. 1993. La Mesure des Arbres et des Peuplements Forestiers. Edition Presses Agronomiques de Gembloux : Gembloux.

Saint-André L, Thongo MB A, Mabiala A, Mouvondy W, Jourdan C, Roupsard O, Deleporte P, Hamel O, Nouvellon Y. 2005. Age-related equa- tions for above- and belowground biomass of a Eucalyptus hybrid in Congo. Forest Ecology and Management, 205(1-3): 199-214.

Soumaré A. 1992. Evolution géomorphologique récente des paysages du Bas-Saloum. Mémoire de DEA, Univ. Cheikh Anta Diop de Dakar, p61. 